\title{
Free-space-to-waveguide demultiplexer featuring zero-contrast gratings
}

\author{
Yin Xu, Xumin Gao, Dan Bai, Guixia Zhu, Jialei Yuan, Hongbo Zhu, and Yongjin Wang* \\ Grünberg Research Center, Nanjing University of Posts and Telecommunications, Nanjing 210003, China \\ E-mail: wangyj@njupt.edu.cn
}

\begin{abstract}
We present here a method to achieve an optical demultiplexer working in the visible range to achieve either a polarization beamsplitter (PBS) or the wavelength-division multiplexer (WDM), which is based on the integration of different periodic gratings using the suspended zero contrast grating (ZCG) structure. The proposed demultiplexer comprises two independent $\mathrm{ZCG} \mathrm{HfO}_{2}$ filters, which are very close to each other so that they can be illuminated and measured at the same time. By combining two independent $\mathrm{HfO}_{2} \mathrm{ZCG}$ filters with selectable functionalities in parallel and extending the waveguides on both sides, a PBS device or WDM device can be achieved. The finite difference time-domain (FDTD) method is used to simulate the coupling properties of the ZCG filters. The simulation results show that the proposed PBS device can effectively direct different polarized light with a wavelength of $650 \mathrm{~nm}$ into separate waveguides. The WDM device can also separate incoming $550 \mathrm{~nm}$ and $650 \mathrm{~nm}$ light beams into separate waveguides. The fabricated devices are characterized by the optical microscope and the atomic force microscope (AFM).
\end{abstract}

Index Terms: Waveguides, planar; Integrated optics devices; Nanostructure fabrication.

\section{Introduction}

Integrated photonic devices play a significant role in various fields, and the coupling between free-space-optics and in-plane photonic circuits is a challenging issue [1-3]. Extensive research has been conducted, especially for telecommunications applications [4-9]. The overlap between the periodic grating fields and the waveguide propagation mode enables resonant gratings to couple the normal-incident light into an in-plane waveguide with high efficiency [10]. Because the guided modes of the gratings are supportable by the in-plane waveguides, they can propagate steadily along the waveguides. By integrating different gratings on one chip, different modes guided by the gratings can be coupled into separate waveguides, which is the main idea of the optical demultiplexer. A variety of designs have been proposed to realize free-space-to-waveguide optical demultiplexers, mainly focusing on the infrared range. Zhu et al. demonstrated a subwavelength high-contrast grating (HCG) to achieve vertical to in-plane optical coupling with high efficiency $[11,12]$. In addition to providing an interconnect between free-space and on-chip photonic circuits, various couplers have also been reported to manipulate light during optical interconnects. Guo et al. demonstrated a plasmonic nanoantenna to direct light of two wavelengths in opposite directions [13]. Fashioned with the inverse-design concept, Piggott et al. presented wavelength demultiplexers with high coupling efficiencies, which can separate $1300 \mathrm{~nm}$ and $1550 \mathrm{~nm}$ light into opposite directions of a silicon waveguide [14, 15].

In this paper, we propose a free-space-to-waveguide demultiplexer featuring zero-contrast grating (ZCG) filters using $\mathrm{HfO}_{2}$ material, which works in the visible wavelength range. The combination of 
different independent ZCG filters with selectable functionalities can filter either the polarization or wavelength of the incident light to realize a polarization beamsplitter (PBS) device or the wavelength-division multiplexer (WDM) device, respectively.

\section{Simulation and discussion}

\section{2. $1 \mathrm{ZCG} \mathrm{HfO}_{2}$ filter}

Compared with HCG filters, ZCG filters are also capable of obtaining strong resonances and can eliminate reflections or phase changes upon transition from the grating ridges into the waveguide layer [16]. As an excellent optical material, $\mathrm{HfO}_{2}$ is transparent from the visible to infrared range $[17,18]$ and often acts as a high refractive index material or the waveguide layer in optical devices [19]. The structure of suspended $\mathrm{HfO}_{2}$ ZCG filters has been demonstrated in the visible wavelength range [20-22]. Fig. 1 shows the geometry of the proposed $\mathrm{ZCG} \mathrm{HfO}_{2}$ filter, which consists of subwavelength periodic $\mathrm{HfO}_{2}$ gratings and a homogeneous $\mathrm{HfO}_{2}$ layer. The silicon substrate underneath the PBS device is removed; thus, the $\mathrm{HfO}_{2} \mathrm{ZCG}$ filter is freestanding. The main parameters of the filter include the grating period $\Lambda$, grating width $w$, the refractive index of the $\mathrm{HfO}_{2}$ film $n_{\mathrm{HfO} 2}$, grating thickness $t_{g}$, waveguiding layer thickness $t_{w}$ and total thickness of $\mathrm{HfO}_{2}$ film $t_{m}$, where $t_{m}=t_{w}+t_{g}$.

By extending the waveguides at both sides of the ZCG, only the resonant modes that are supported by the waveguides can stably propagate along the waveguides and be detected at the output facets. To maximize the coupling between the incoming light and the ZCG filter, the device parameters are optimized using the finite-difference time-domain (FDTD) method [23] with a static refractive index of $\mathrm{HfO}_{2}, n_{\mathrm{HFO} 2}=2.05$. The filling factor $\eta$ refers to the ratio of the grating width $\mathrm{w}$ with respect to the grating period $\Lambda$, namely, $\eta=w / \Lambda$. The coupling efficiency is defined as the intensity of light detected at the output facets of the waveguides on either side with respect to that of the incident intensity. In the simulation, the Gaussian incident beam with $10 \mu \mathrm{m}$ in diameter is normally illuminated onto the proposed ZCG filter. As Shown in Figs. 2(a) and 2(b), optimizations of the grating period $\Lambda$ and the filling factor $\eta$ are conducted by fixing $t_{g}=0.07 \mu \mathrm{m}$ and $t_{w}=0.13 \mu \mathrm{m}$, under TE and TM polarization respectively. The results show that the device achieves the highest coupling efficiency for TE polarization at the grating period $\Lambda=0.39 \mu \mathrm{m}$ and filling factor $\eta=0.45$ while for TM polarization, the device exhibits the best coupling performance at the combination of $\Lambda=0.49 \mu \mathrm{m}$ and $\eta=0.5$. For a given $\mathrm{HfO}_{2}$-on-silicon platform, the total thickness of the $\mathrm{HfO}_{2}$ film is fixed. Hence, the thickness of the $\mathrm{HfO}_{2}$ waveguiding layer decreases as the $\mathrm{HfO}_{2}$ grating thickness increases. There is a trade-off between the thicknesses of the $\mathrm{HfO}_{2}$ grating layer and the $\mathrm{HfO}_{2}$ waveguiding layer where the optimal optical response can be obtained. Based on the FDTD method, the thicknesses of the $\mathrm{HfO}_{2}$ grating and the $\mathrm{HfO}_{2}$ waveguide layer are optimized to achieve the highest coupling efficiency, which is illustrated in Fig. 2(c). The coupling efficiency spectra versus the grating thickness $t_{g}$ is simulated with $\Lambda=0.39 \mu \mathrm{m}$ and $\eta=0.45$ under TE polarization, and with $\Lambda=0.49 \mu \mathrm{m}$ and $\eta=0.5$ under TM polarization, where the total thickness of $\mathrm{HfO}_{2}$ film $t_{m}$ is $200 \mathrm{~nm}$. The two $\mathrm{ZCG} \mathrm{HfO}_{2}$ filters share the same coupling peak location at $\mathrm{t}_{\mathrm{g}}=0.07 \mu \mathrm{m}$; thus, the $\mathrm{HfO}_{2}$ waveguiding layer thickness $t_{w}=\left(t_{m}-t_{g}\right)=0.13 \mu \mathrm{m}$, where low sideband and narrow line-width can be achieved, laying the basis for integrating different $\mathrm{ZCG} \mathrm{HfO}_{2}$ filters on one chip.

\section{2 PBS featuring $\mathrm{ZCG} \mathrm{HfO}_{2}$ filters}

Based on the FDTD method, Figs. 3 demonstrates the optical responses of the single $\mathrm{ZCG}_{\mathrm{HfO}}$ filters operating at the wavelength of $650 \mathrm{~nm}$. With the dimensions set at $\Lambda=0.39 \mu \mathrm{m}$ and $\eta=0.45$ and the number of grating bars at 40, Fig. 3(a) illustrates the amplitude of electric field for the ZCG filter operating at $650 \mathrm{~nm}$ for TE polarization. When the light is TE-polarized, incident light can be effectively coupled into the grating and laterally propagates along the in-plane waveguides. However, the coupling for TM-polarized light is weak, as shown in Fig. 3(b). Due to overlap effect of the coupled light, the field intensity in specific areas in the waveguide may exceed unit intensity, which causes white spots in the field profiles. The simulated coupling efficiency spectra are shown in Fig. 3(c). For TE-polarized light, 
the simulated coupling efficiency into the grating is 0.237 at $650 \mathrm{~nm}$, whereas for TM-polarized light at $650 \mathrm{~nm}$, the efficiency is negligible. For the ZCG filter with $\Lambda=0.49 \mu \mathrm{m}, \eta=0.5$ and 32 grating bars, the TM-polarized incident light at $650 \mathrm{~nm}$ can be coupled into the grating effectively and propagates along the in-plane waveguides laterally, as plotted in Fig. 3(e), whereas the coupling for TE-polarized light is weak, as shown in Fig. 3(d). Correspondingly, the simulated coupling efficiency spectra are demonstrated in Fig. 3(f). Hence, for the free-space-to-waveguide demultiplexer case, the polarization splitter can be achieved by putting such two separate ZCG filters side by side. The normal-incident light is coupled into the gratings and then propagates along the in-plane waveguides in different directions with different light coupling efficiencies.

The proposed PBS device featuring $\mathrm{ZCG} \mathrm{HfO}_{2}$ filters is based on a parallel combination of two ZCG $\mathrm{HfO}_{2}$ gratings with different parameters, and the waveguides at both sides are extended to achieve stable propagation, which is schematically shown in Fig. 4. The optical behavior of the $\mathrm{HfO}_{2} \mathrm{ZCG}$ filter is

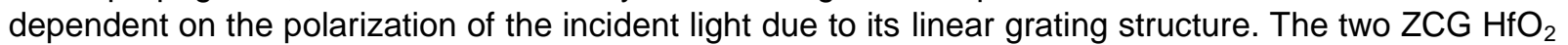
filters are close to each other so that they can be illuminated and measured at the same time. When the polarized light is incident on the device, the maximum couplings between the incoming light and the two ZCG filters occur at different propagation directions due to the different polarizations of the incoming light, leading to the polarization-sensitive performance. Realized on the same chip with the same fabrication process, the two independent ZCG filters share the same grating thickness $t_{g}=0.07 \mu \mathrm{m}$ and waveguide layer thickness $t_{w}=0.13 \mu \mathrm{m}$.

For the incident light with a wavelength of $650 \mathrm{~nm}$, the dimensions of the ZCG filters making up the PBS are optimized as $\Lambda_{1}=0.39 \mu \mathrm{m}, \eta_{1}=0.45$ with 19 grating bars and $\Lambda_{2}=0.49 \mu \mathrm{m}, \eta_{2}=0.5$ with 15 grating bars, according to the above analysis. The field profiles of the proposed PBS device are shown in Figs. 5, where Fig. 5(a) refers to the TE polarized incidence and Fig. 5(b) to TM. TE-polarized light is coupled into the left waveguide and can be detected at the left output facet (Port 1) whereas TM-polarized light is coupled into the right waveguide and can be detected at the right output facet (Port 2). In Fig. 5(a), the coupling efficiencies at the left side and the right side are $10.1 \%$ and $2.2 \%$, respectively, whereas in Fig. $5(b)$, they are $1.0 \%$ and $7.1 \%$, respectively.

\section{3 WDM featuring $\mathrm{ZCG} \mathrm{HfO}_{2}$ filters}

Because the optical behavior of the ZCG filter is dependent on the grating period, the pitch-sensitive coupling can realize multi-channel wavelength demultiplexing. In a similar method, the proposed WDM grating is based on a parallel combination with two independent $\mathrm{HfO}_{2} \mathrm{ZCG}$ filters with different periods $\left(\Lambda_{3}, \Lambda_{4}\right)$ and different widths $\left(w_{3}, w_{4}\right)$, which is schematically shown in Fig. 6 . The two ZCG filters are close to each other and can separate different wavelengths of the incident light into separate waveguides in different directions when the device is illuminated. The light coupling efficiency is dependent on the structural parameters of the $\mathrm{HfO}_{2} \mathrm{ZCG}$ filter. Putting two different the $\mathrm{HfO}_{2} \mathrm{ZCG}$ filters together, the integrated the $\mathrm{HfO}_{2} \mathrm{ZCG}$ filters will have selectable coupling efficiency, leading to a high output in one direction and a weak output in opposite direction. Hence, the integrated the $\mathrm{HfO}_{2} \mathrm{ZCG}$ filters can be used as a wavelength-division multiplexer. By making the peak coupling at 550nm, the dimensions for the left ZCG filter are optimized as $\Lambda_{3}=0.32 \mu \mathrm{m}$ and $\eta_{3}=0.42$ with 23 grating bars, whereas, for the right filter, the values are designed as $\Lambda_{4}=0.39 \mu \mathrm{m}$ and $\eta_{4}=0.45$ with 19 grating bars for the peak coupling at $650 \mathrm{~nm}$, which is based on the FDTD analysis.

The coupling properties of the proposed WDM device are simulated by the FDTD method. The predominant coupling of the incident light occurs at the left side, as can be distinctly observed from Fig. $7(\mathrm{a})$, which illustrates the amplitude of electric field for the WDM operating at $550 \mathrm{~nm}$ for TE polarized light. In comparison, the incident light at $650 \mathrm{~nm}$ with TE polarization is predominantly coupled into the gratings on the right side, as clearly shown in Fig. 7(b). The simulated coupling efficiency spectra of the WDM device is plotted in Fig. 7(c), which shows that at $550 \mathrm{~nm}$, the simulated coupling efficiencies at the left and right output facets of the waveguides are $11.0 \%$ and $2.9 \%$, respectively, whereas at $650 \mathrm{~nm}$, the 
coupling efficiencies are $1.3 \%$ and $12.5 \%$, respectively. The splitting ratio, defined as the ratio of the power emitted from the two output ports, is plotted in Fig. 6(d). The proposed WDM grating has a calculated splitting ratio of $5.79 \mathrm{~dB}$ at $550 \mathrm{~nm}$ and $9.67 \mathrm{~dB}$ at $650 \mathrm{~nm}$, respectively. Additionally, the general strategy of a free-space-to-waveguide demultiplexer featuring ZCGs with different design parameters can be extended to support a larger number of wavelength channels by integrating more ZCG filters at a higher density.

\section{Device fabrication}

The proposed integrated photonic devices are implemented on an $\mathrm{HfO}_{2}$-on-silicon platform. Both $\mathrm{HfO}_{2}$ rib waveguides and $\mathrm{HfO}_{2}$ gratings are defined by electron beam lithography and formed by ion beam etching. Silicon removal is then conducted to generate freestanding device architecture, which are suspended by support beams. The $\mathrm{HfO}_{2}$ rib waveguides impinge on the $\mathrm{HfO}_{2}$ gratings to couple the diffracted light. When the incident light illuminates on the $\mathrm{HfO}_{2} \mathrm{ZCG}$ filter, the light is diffracted and coupled into the $\mathrm{HfO}_{2}$ rib waveguides. Fig. 8(a) shows an optical micrograph image of the PBS device, which is fabricated on suspended $\mathrm{HfO}_{2}$ membrane. The ZCG filter is connected to a waveguide, which ends with a finite length. Therefore, the coupled light in the waveguide is diffracted into air at the abrupt air/ $/ \mathrm{HfO}_{2}$ end facet, leading to a direct observation of light coupling between ZCG filter and waveguide. For practical use, it is feasible to design and achieve the light coupling between single mode waveguide and ZCG filter on a 200-nm-thick $\mathrm{HfO}_{2}$ film with the introduction of a tapered waveguide. In Figs. 8(b) and (c), an atomic force microscope is used to characterize the surface profile and etching depth of the device. The etching profiles of the two ZCG filters with different parameters (white box in Fig. 8(b)) are plotted in Fig. 8(c). The thickness of the grating layer is $70 \mathrm{~nm}$ and the periods of the two gratings are $0.39 \mu \mathrm{m}$ for the left and $0.49 \mu \mathrm{m}$ for the right, respectively. The device is fabricated on the 200-nm-thick suspended $\mathrm{HfO}_{2}$ membrane as designed, indicating that the proposed device configuration is feasible to manufacture.

\section{Summary}

We have presented a method to achieve optical demultiplexers working in the visible range to realize

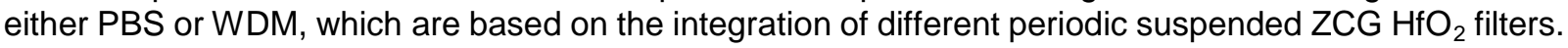
Specifically, by parallel combination of two independent $\mathrm{ZCG} \mathrm{HfO}_{2}$ filters with selectable functionalities, a PBS device or WDM device can be achieved. The coupling properties of the ZCG filters are simulated by the FDTD method. The proposed PBS device featuring $\mathrm{ZCG} \mathrm{HfO}_{2}$ filters can effectively separate TEand TM-polarized light with a wavelength of $650 \mathrm{~nm}$ into separate waveguides. The WDM device featuring ZCG filters is demonstrated to divide the incoming TE-polarized $550 \mathrm{~nm}$ and $650 \mathrm{~nm}$ light beams into separate waveguides. The optical microscope and the atomic force microscope are used to characterize the fabricated devices. Flexibly, based on the general strategy of integrating more ZCG $\mathrm{HfO}_{2}$ filters with certain parameters on one chip, the free-space-to-waveguide demultiplexer featuring zero-contrast gratings can be achieved to realize multi-channel PBS or WDM devices.

\section{Acknowledgements}

This work is jointly supported by the NSFC (11104147, 61322112) and research projects (2014CB360507, RLD201204, BJ211026, SJZZ_0105).

\section{References}

[1] C. Kopp, S. Bernabé, B. B. Bakir, J. M. Fedeli, R. Orobtchouk, F. Schrank, H. Porte, L. Zimmermann, and Tolga Tekin, 2011 "Silicon photonic circuits: on-CMOS integration, fiber optical coupling, and packaging," IEEE J Sel Top Quant 17(3), 498-509.

[2] J. V. Roey, B. Denturck, and P. E. Lagasse, "Guided wave coupling in integrated-optic gratings: normal incidence," IEE P. H 
131(5), 282-289 (1984).

[3] A. S. Barker, Jr.,“Direct optical coupling to surface excitations,” Phys. Rev. Lett. 28(14), 892-895 (1972).

[4] H. Yamada, M. Nozawa, M. Kinoshita, and K. Ohashi, "Vertical-coupling optical interface for on-chip optical interconnection," Opt. Express 19(2), 698-703 (2011).

[5] M. Haurylau, G. Chen, H. Chen, J.Zhang, N. A. Nelson, D. H. Albonesi, E. G. Friedman, and P. M. Fauchet, "On-chip optical interconnect roadmap: Challenges and critical directions," IEEE J Sel Top Quant 12(6), 1699-1705 (2006).

[6] B. Wang, J. Jiang, and G. P. Nordin, "Compact slanted grating couplers," Opt. Express 12(15), 3313-3326 (2004).

[7] G. Marie, L. Vivien, G. Sattler, A. Kazmierczak, B. Sanchez, K. B. Gylfason, A. Griol, D. Marris-Morini, E. Cassan, D. Giannone, H. Sohlström, and D. Hill, "High efficiency silicon nitride surface grating couplers," Opt. Express 16(1), 328-333 (2008).

[8] G. Roelkens, D. Vermenlen, F. V. Laere, S. Selvaraja, S. Scheerlinck, D. Taillaert, W. Bogaerts, P. Dumon, D. V. Thourhout, and R. Baet, "Bridging the gap between nanophotonic waveguide circuits and single mode optical fibers using diffrative grating structure," J. Nanosci. Nanotechnol. 10(3), 1551-1562 (2010).

[9] D. Taillaert, H. Chong, P. I. Borel, L. H. Frandsen, R. M. De La Rue, and R. Beats, "A compact two-demensional grating coupler used as a polarization splitter," IEEE Photon. Technol. Lett. 31(24), 2097-1251 (2003).

[10] S. S. Wang, R. Magnusson, "Theory and applications of guided-mode resonance filters," Appl. Opt. 32(14), 2606-2613 (1993).

[11] L. Zhu, V. Karagodsky, W. Yang, and C. Chang-Hasnain,"Novel high efficiency vertical optical coupler using subwavelength high contrast grating," in Conference on Lasers and Electro-Optics/Laser Applications to Photonic Applications, Technical

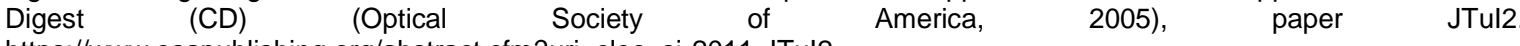
https://www.osapublishing.org/abstract.cfm?uri=cleo si-2011-JTul2

[12] L. Zhu, V. Karagodsky, and C. Chang-Hasnain,"Novel high efficiency vertical to in-plane optical coupler," Proc. SPIE 8270, 82700L1-11(2012).

[13] R. Guo, M. Decker, F. Setzpfandt, I. Staude, D. N. Neshev, and Y. S. Kiveshar, "Plasmonic fano nanoantennas for on-chip seprartion of wavelength-encoded optical signals," Nano Lett. 15, 3324-3328 (2015).

[14] A. Y. Piggott, J. Lu, T. M. Babinec, K.G. Lagoudakis, J. Petykiewicz, and J. Vuckovic, "Inverse design and implementation of a wavelength demultiplexing grating coupler," Sicentific Reports 4, 7210 (2014).

[15] A. Y. Piggott, J. Lu, K.G. Lagoudakis, J. Petykiewicz, T. M. Babinec, and J. Vuckovic, "Inverse design and demonstration of a compact and broadband on-chip wavelength demultiplexer," Nature. Phot. 9, 374-377 (2015).

[16] R. Magnusson, "Wideband reflectors with zero-contrast gratings," Opt. Lett. 39(15),4337-4340 (2014).

[17] Y. Wang, X. Gao, Z. Shi, L. Chen, M. L. Garcia, N. A. Hueting, M. Cryan, X. Li, M. Zhang, and H. Zhu, "Guided-Mode Resonant $\mathrm{HfO}_{2}$ Grating at Visible Wavelength Range," IEEE Photon.J. 6, 2200407 (2014).

[18] Y. Wang, T. Wu, Y. Kanamori, and K. Hane, "Freestanding $\mathrm{HfO}_{2}$ grating fabricated by fast atom beam etching," Nanoscale Res. Lett. 6, 367(2011).

[19] S. Liu,Y.Jin,Y. Cui,J.Ma, J. Shao, and Z. Fan, "Characteristics of high reflection mirror with an $\mathrm{SiO}_{2}$ top layer for multilayer dielectric grating," J. Phys.D. 40, 3224 (2007)

[20] M. J. Uddin, T. Khaleque, and R. Magnusson, "Guided-mode resonant polarization-controlled tunable color filters," Opt. Express 22(10), 12307-12315 (2014).

[21] X. Gao, Z. Shi, X. Li, H. Zhu, and Y. Wang, "Multiline resonant filters fashioned with different periodic subwavelength gratings," Opt. Lett. 39(23), 6660-6663 (2014).

[22] X. Gao, T. Wu, Y. Xu, X. Li, D. Bai, G. Zhu, H. Zhu, and Y. Wang, "Angular-dependent polarization-insensitive filter fashioned with zero-contrast grating," Opt. Express 23(12), 15235-15241 (2015).

[23] J.P. Berenger, "A perfectly matched layer for the absorption of electromagnetic waves," J. Comput. Phys. 114, 185-200 (1994). 


\section{Illustration Captions}

Fig. 1. Structure of the suspended $\mathrm{HfO}_{2} \mathrm{ZCG}$ filter.

Fig. 2. Calculated coupling efficiency versus filling factor $\eta$ and grating period $\wedge$ when the incident wavelength is $650 \mathrm{~nm}$ and with $t_{g}=0.07 \mu \mathrm{m}$ and $t_{w}=0.13 \mu \mathrm{m}$ under (a) TE polarization; (b) TM polarization. (c) Coupling efficiency spectra versus the grating thickness $t_{g}$ with $\Lambda=0.39 \mu \mathrm{m}$ and $\eta=0.45$ under TE polarization and $\Lambda=0.49 \mu \mathrm{m}$ and $\eta=0.5$ under TM polarization.

Fig. 3. The amplitudes of electric field for the ZCG filter with $\Lambda=0.39 \mu \mathrm{m}$ and $\eta=0.45$ operating at $650 \mathrm{~nm}$ under (a) TE polarization, (b) TM polarization and (c) the corresponding coupling efficiency spectra; Field profiles of the ZCG filter with $\Lambda=0.49 \mu \mathrm{m}$ and $\eta=0.5$ operating at $650 \mathrm{~nm}$ under (d) TE polarization, (e) TM polarization and (f) the corresponding coupling efficiency spectra.

Fig. 4. Schematic of the PBS device featuring $\mathrm{ZCG} \mathrm{HfO}_{2}$ filters.

Fig. 5. The amplitudes of electric field for proposed PBS device featuring featured with $\mathrm{ZCG} \mathrm{HfO}_{2}$ filters when the incident light is $650 \mathrm{~nm}$ under (a) TE polarization and (b) TM polarization. TE-polarized light is coupled into the left waveguide and can be detected at Port 1, whereas TM polarized light is coupled into the right waveguide and can be detected at Port 2.

Fig. 6. Schematic of the WDM device featuring suspended $\mathrm{ZCG} \mathrm{HfO} 2$ filters.

Fig. 7. The amplitudes of electric field for the WDM device under (a) TE-polarized $550 \mathrm{~nm}$ illumination; (b) TE-polarized $650 \mathrm{~nm}$ illumination; (c) Coupling efficiency spectra of the WDM device; (d) the corresponding splitting ratio spectra.

Fig. 8. (a) Optical micrograph image of the PBS device; (b) Atomic force microscope image of the PBS device; (c) Etching depth of the zero-contrast gratings. 


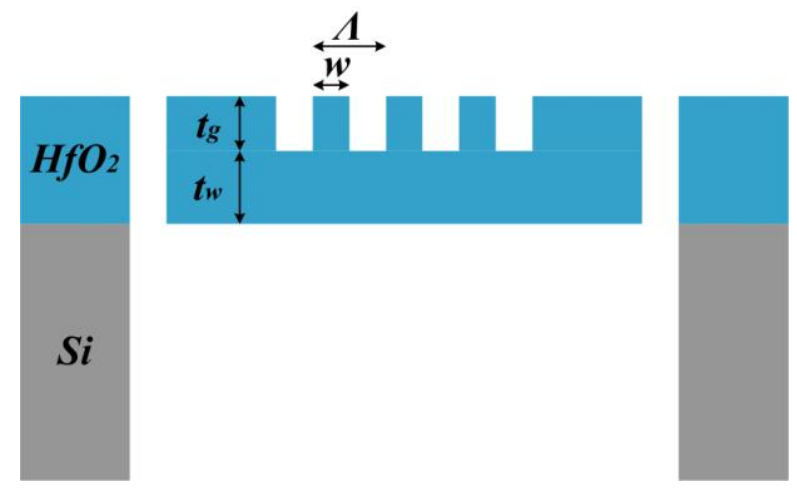

Figure 1

Yin Xu et al. 


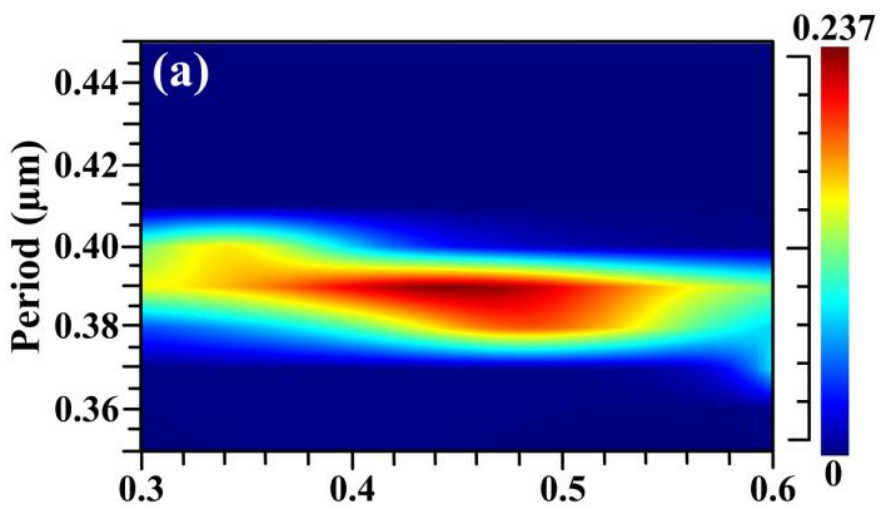

Filling Factor
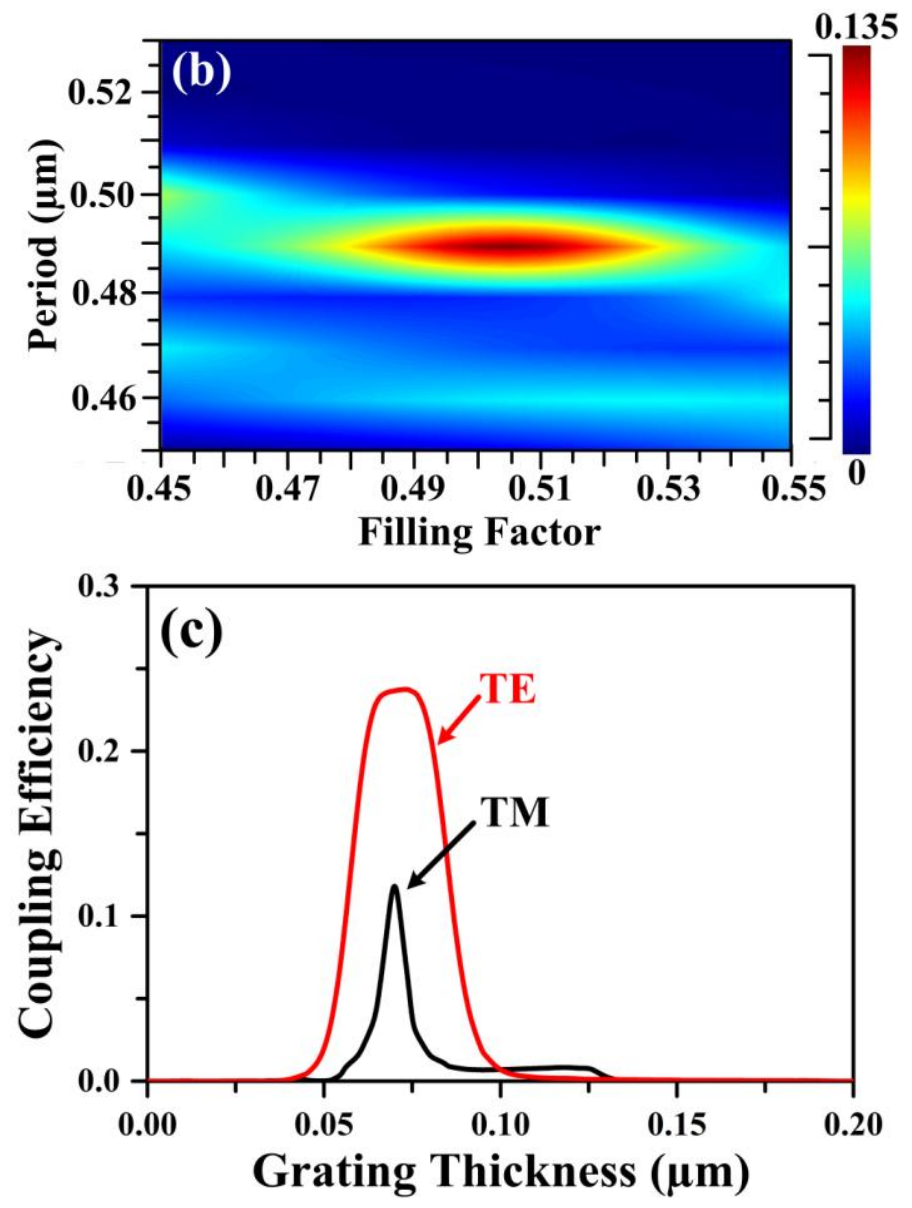

Figure 2

Yin $\mathrm{Xu}$ et al. 

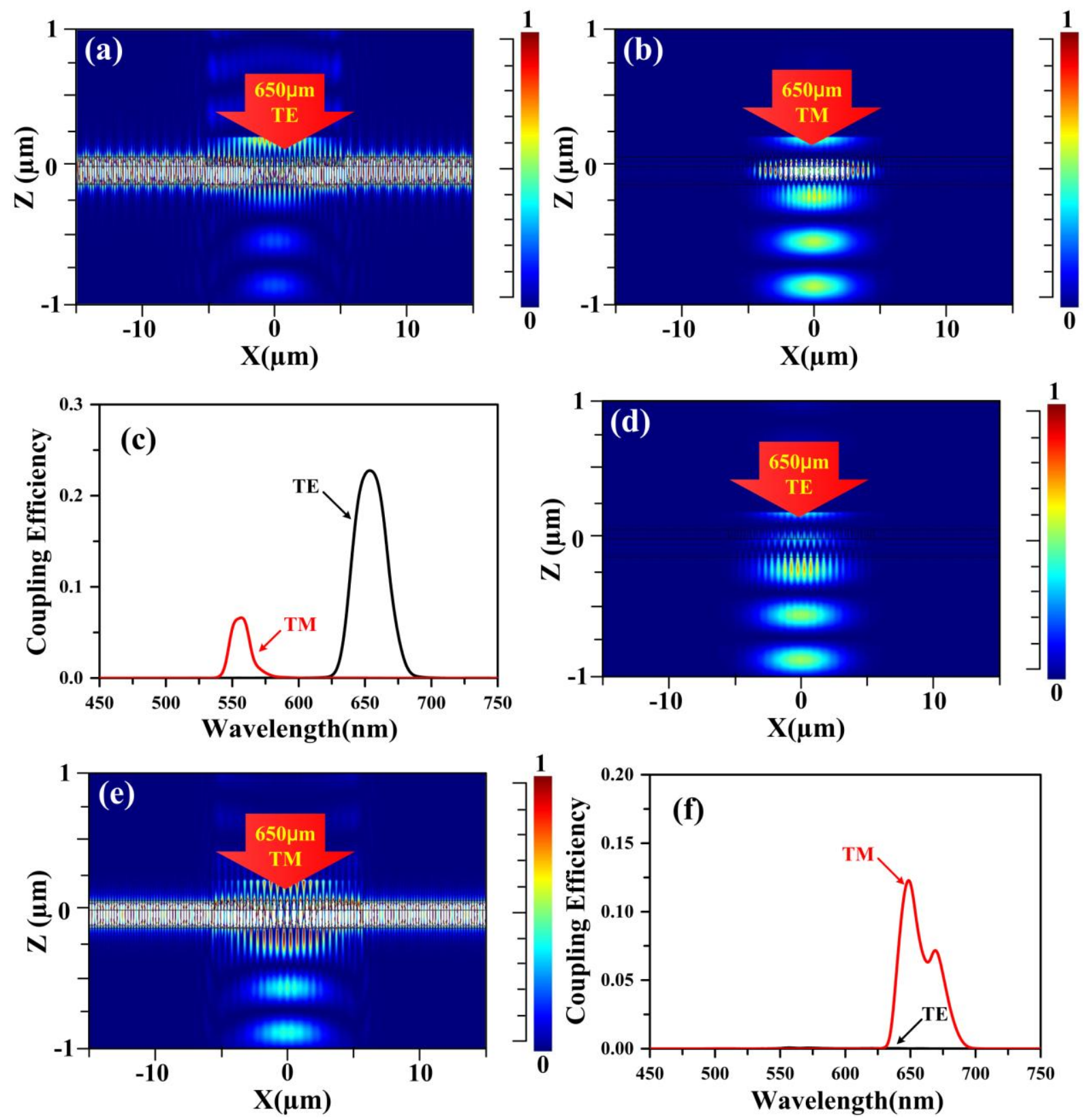

Figure 3

Yin Xu et al. 


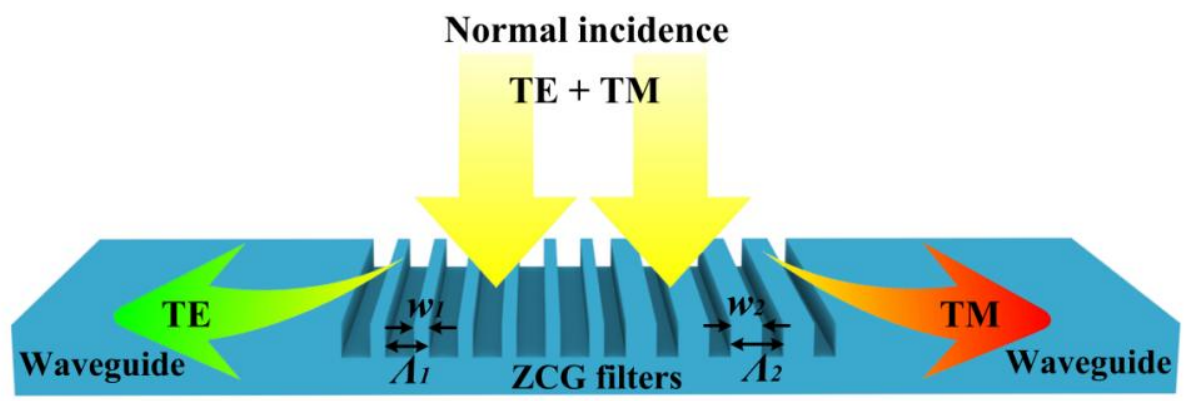

Figure 4

Yin Xu et al. 

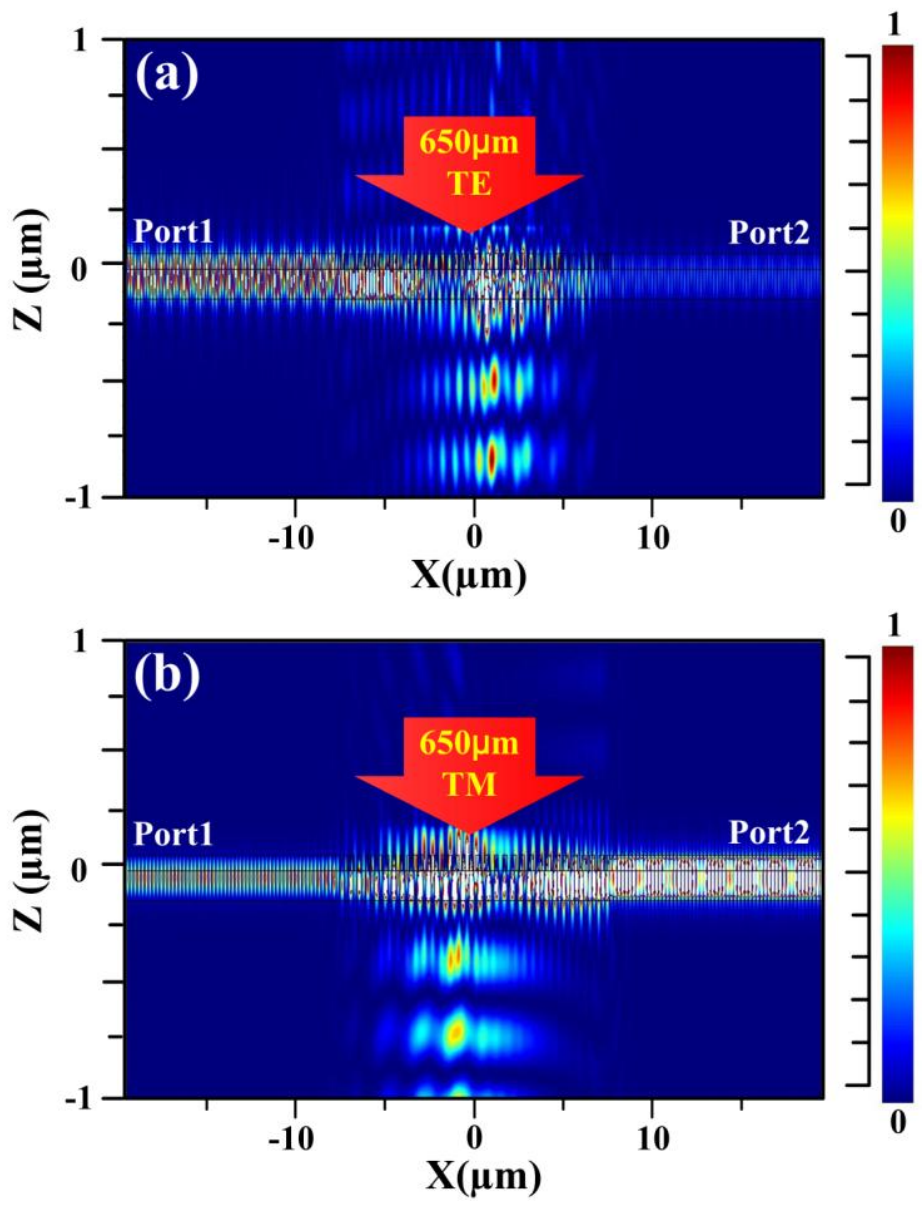

Figure 5

Yin $\mathrm{Xu}$ et al. 


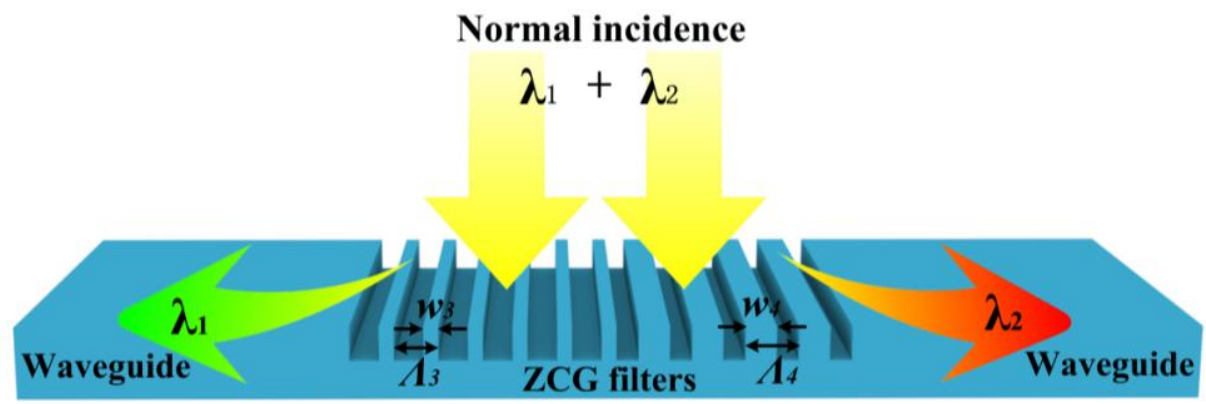

Figure 6

Yin $\mathrm{Xu}$ et al. 

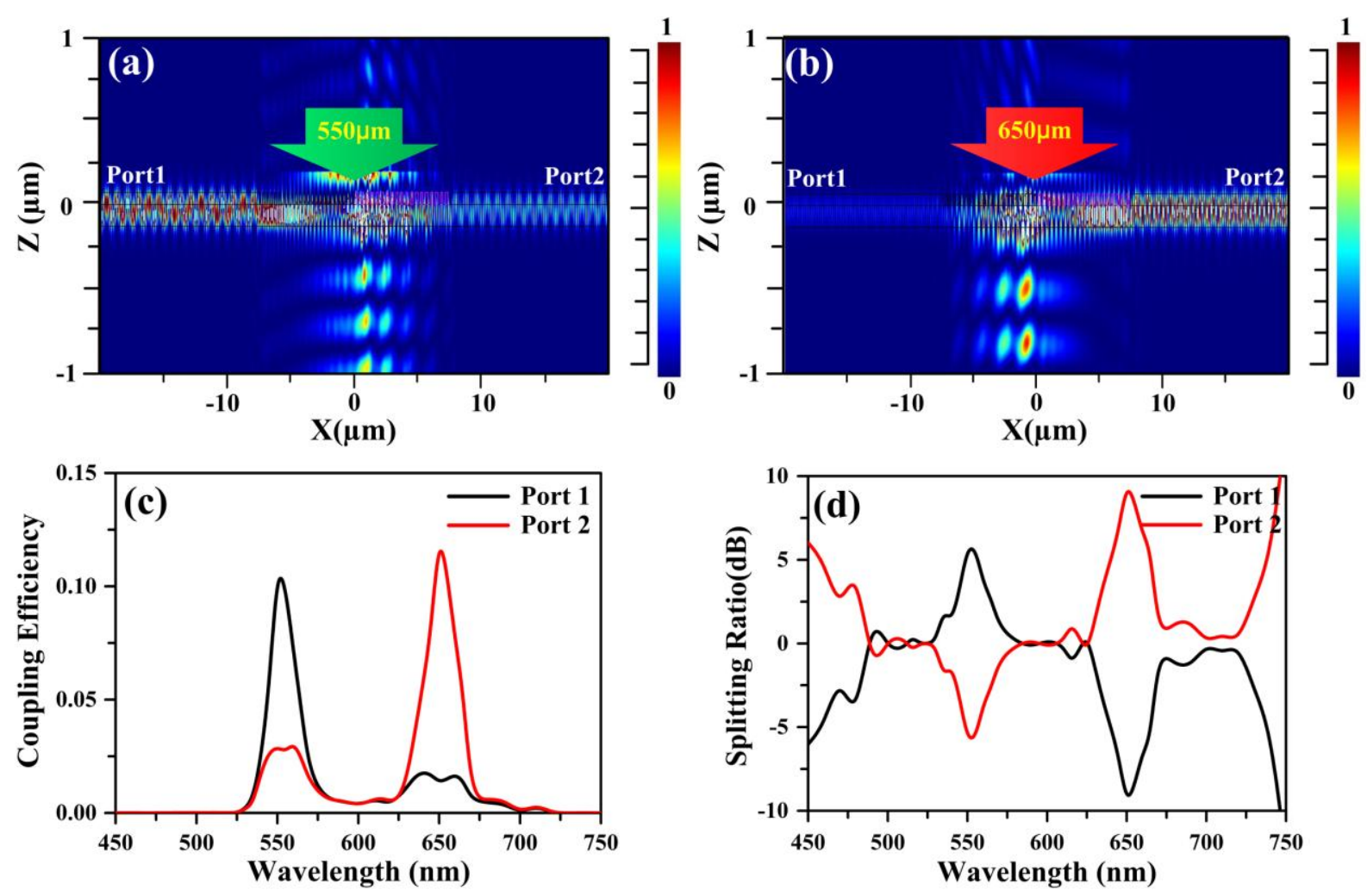

Figure 7

Yin $\mathrm{Xu}$ et al. 

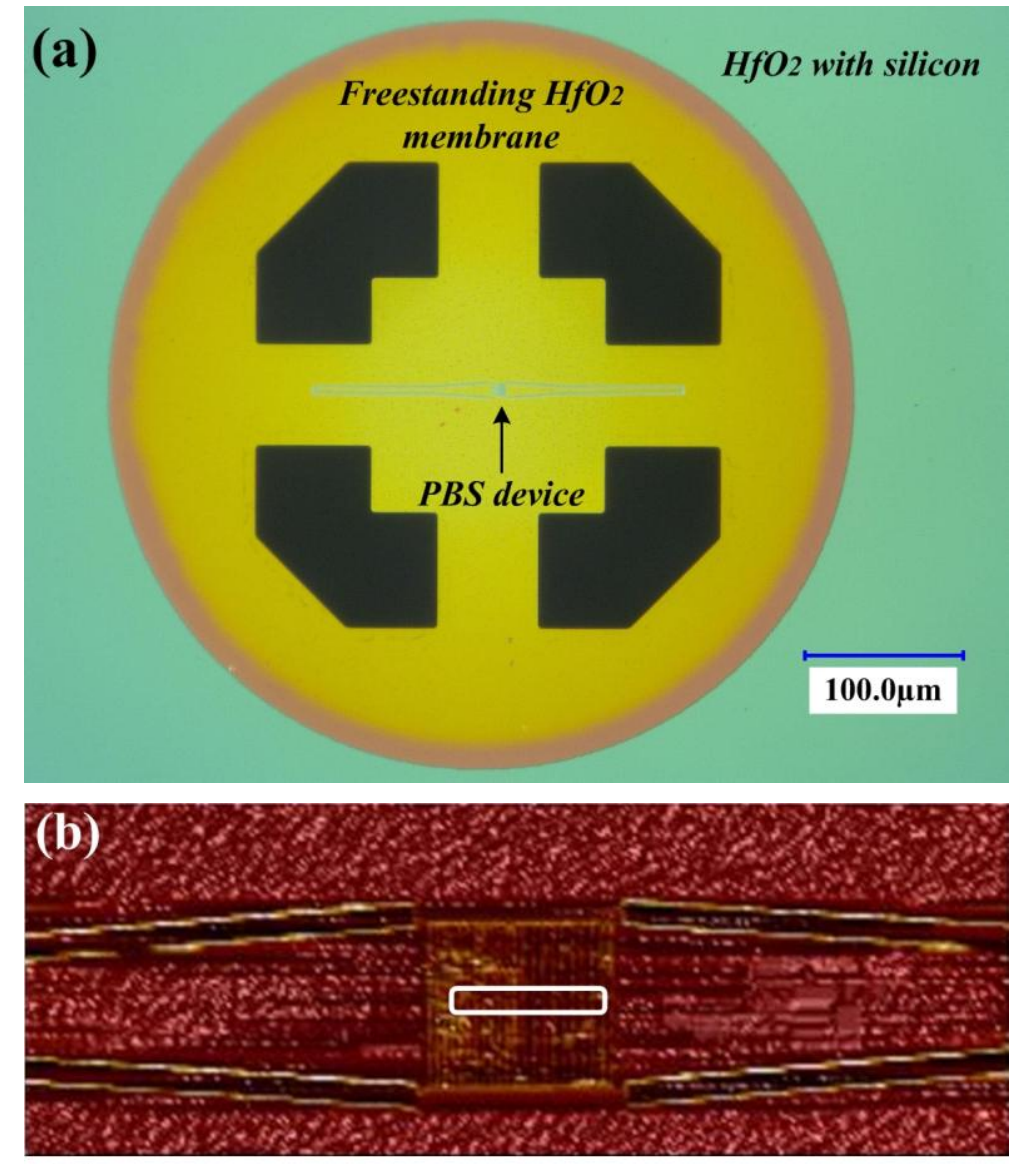

(c)

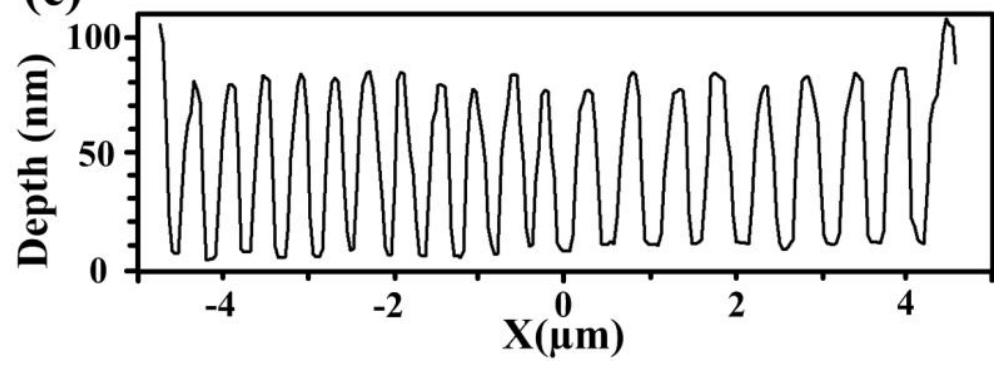

Figure 8

Yin $\mathrm{Xu}$ et al. 